\title{
Leptotrichia amnionil: certain pathogen in pyosalpinx
}

\author{
Sandro Pierdomenico, Andreina Baj, Giuseppe Massari, Alberto Colombo, Sergio Gallazzi \\ Laboratorio di Microbiologia, A.O. Ospedale di Circolo e Fondazione Macchi, Varese
}

Key words: Anaerobes, Leptotrichia amnionii, Pyosalpinx, Pelvic Inflammatory Disease

\section{SUMMARY}

Leptotrichia amnionii has had a recent taxonomic definition (2002) and definitely belongs to fastidious Gram negative anaerobes group thanks to difficulties to culture and preserve. Few PubMed reports of microbiological detections are documented to date and this is the first isolation described in Italy. Frequently only DNA analysis in direct samples succeeds in finding it out. Our study describes a case of Leptotrichic amnionii successfully cultivated from aspirated pus, but only I6S rRNA gene amplification and subsequent sequencing technique could afford to identify. The work remarks the added value of DNA techniques in routine analysis of anaerobes.

\section{INTRODUCTION}

On $12^{\text {th }}$ December 2009, a 53 years-old-female was admitted to gynecological ward for recurrent abdominal pain, amenorrhea and uterine bleeding, with a history of similar events since November $11^{\text {th }}$, treated by GP with analgesics. Clinical examination showed multiple nodular uterine fibromatosis in a patient with previous thyroidectomy for goiter, under L-thyroxine treatment, as unique concomitant disease. No further investigations were performed and no treatment prescribed awaiting for a $2^{\text {nd }}$ check on $18^{\text {th }}$ January 2010.

Suddenly on $4^{\text {th }}$ January the patient reached emergency ward for acute abdominal pain, fever and massive vaginal bleeding. The acute inflammatory state was documented by blood data: $\mathrm{WBC}=23.3 \times 10^{3} / \mathrm{mm}^{3}$ with 18.58 segmented neutrophils, C-reactive protein level $(\mathrm{CRP})=17.6 \mathrm{mg} / \mathrm{L}$ and platelet count up to $797 \times 10^{3} / \mathrm{mm}^{3}$.

Physical examination and vaginal ultrasound contributed to the diagnosis of PID and laparoscopy showed right pyosalpinx.

\section{MATERIALS AND METHODS}

Two fractions of aspirated pus from abscess site, during laparoscopy, were inoculated in both Aerobic and Anaerobic Bactec Plus culture vials (BD, Meylan, France) without any supplement for fastidious organisms (FOS).

Cultural monitoring started on 04 January at 15:40; the positivity was flagged on 07 January 2010 at 20:47 in Anaerobic bottle (lag time $=77$ hours). Subcultures in Blood and Chocolate plates (BD) were performed as usual in anaerobic Jars with sachets (AnaeroGen ${ }^{\mathrm{TM}}$, Oxoid Ltd., Basingstoke, England). Initial growth was observed after 48 hours: only few, slight gray, $1 \mathrm{~mm}$ tiny convex and lightly moist colonies were detectable by dissecting microscope.

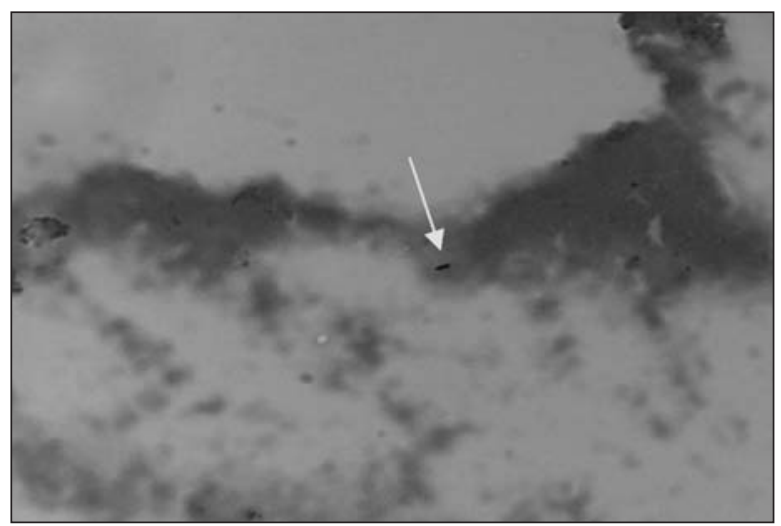

Figura I. Gram staining.
Gram staining showed weak positivity for non spore forming, regular body, pleomorphic rods and coccobacilli, without any granular inclusion or branched shape (Figures I, II). All efforts to enrich the culture were unsuccessful works. The poverty of colonies compelled to leave BBL Crystal System (6 MF necessary) and to choose API ANA (bioMérieux, Marcy l'Etoile, France) to attempt phenotypic identification. After 48 hours of anaerobic incubation only sucrose/glucose had weak fermentative signs. We tried to identify bio-number profile in API WEB Data Base. No available T-index was matched. Anaerobes presence was suggested related to gram staining. We reported it thinking to Actinomyces and preliminary natural drug susceptibility was discussed, awaiting for DNA analysis.

\section{MOLECULAR TECHNIQUES}

Two or three colonies of cultivated bacteria were suspended in $2 \mathrm{ml}$ of distilled water; resulting $4 \mathrm{ml}$ was concentrated to $200 \mu \mathrm{l}$ by centrifugation $(10000 \times g, 5 \mathrm{~min})$ and DNA was extracted with the QIamp DNA extraction Mini Kit (Qiagen, Valencia, CA) according to the manufacturer's instructions.

DNA was amplified targeting a $1450 \mathrm{bp}$ region within the 16S rRNA gene by two primers:

1 -fwd GAGAGTTTGATCCTGGCTCAG, 2- rvs TACGGCTACCTTGTTACGACTT.

Reaction tubes contained $20 \mathrm{nmol}$ of each primer and following reagents: PCR Buffer $1 \mathrm{X}, \mathrm{MgCl}_{2} 1.5 \mathrm{mM}, 0.25 \mathrm{mM}$ of each dNTP, and $2 \mathrm{U}$ of Taq DNA polymerase. Thermal cycler conditions: 10 -min at $95^{\circ} \mathrm{C}, 35$ cycles of $30 \mathrm{~s}$ at $95^{\circ} \mathrm{C}$ followed by $30 \mathrm{~s}$ at $60^{\circ} \mathrm{C}$ and $30 \mathrm{~s}$ at $72^{\circ} \mathrm{C}$; final extension step at $72^{\circ} \mathrm{C}$ for $15 \mathrm{~min}$ was performed

Amplification products were purified with QIAquick PCR Purification Kit (QIAGEN, Tokyo, Japan) and directly sequenced using the Big Dye Terminator v1.1 cycle

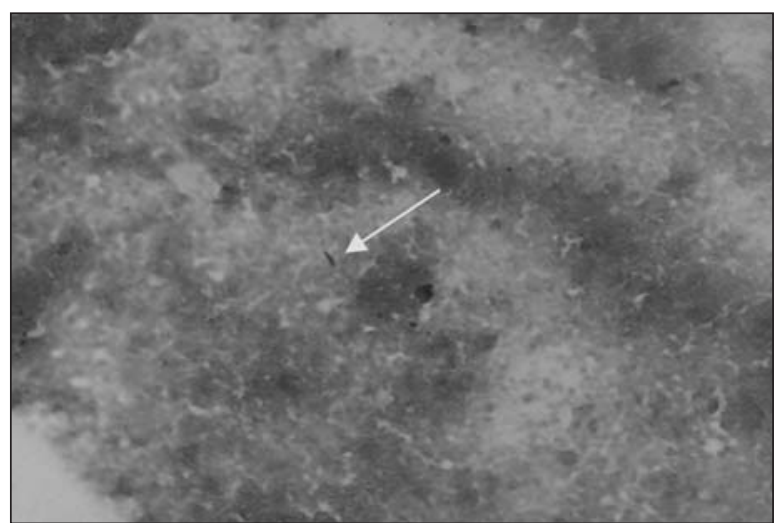

Figura II. Pleomorphism.

Corresponding author: Sandro Pierdomenico

Laboratorio di Patologia Clinica

P.le G. Solaro 3 - 21052 Busto Arsizio (VA) - Tel.: 033 I 699729 - Cell.: +39 33944767 I9

E-mail: spierd@tin.it 
sequencing kit (Applied Biosystems, Foster City, CA) in a final volume of $20 \mu \mathrm{L}$. Forward and reverse primers were used. After purification of sequencing products (Centrisep spin columns; Princeton Separations, Adelphia, NJ), sequencing was performed with an ABI PRISM ${ }^{\mathrm{TM}} 3100$ Genetic analyzer (Applied Biosystems, Foster City, CA).

Nucleotide sequence analysis was carried out using the CLCbio software (Aarhus, Denmark).

The sequence data have been analyzed using CLCbio software and compared with reference sequences on NCBI (National Center for Biotechnology Information) and EMBL
(European Molecular Biology Laboratory).

\section{RESULTS}

DNA analysis provided the presence of a particular anaerobe. Obtained amplicons displayed a similarity of $99 \%(435 / 436)$ and $100 \%(373 / 373)$ with Leptotrichia amnionii (14) (Tables 1, 2: NCBI BLAST - version 2.210).

During pre and post antibiotic regimen no other microbiological findings other than aspirated pus were detected in fluids (blood, urine, peritoneal liquid) or serologically. The patient underwent successful hysterectomy

Table I. Strand Plus/Plus.

16S ribosomal RNA gene, partial sequence Length $=1468$ Score $=800$ bits (433), Expect $=0.0$ Identities $=435 / 436(99 \%)$, Gaps $=0 / 436(0 \%)$

\begin{tabular}{|c|c|c|c|}
\hline Query & GCAAACAACTCTCGTGGTGTGACGGGCGGTGTGTACAAGGCCCG & GTATTCACCGT & 60 \\
\hline & ||Sbjct & & 77 \\
\hline $\mathrm{GCA}$ & GGTGTGACGGGCGGTGTGTACAAGGCCCGAGAACGTATTCACCGT & 136Query & 61 \\
\hline $\mathrm{GACA}$ & CGATTACTAGTGATTCCAACTTCATGAAGTCGAGTTGCAGACTTC & & 120 \\
\hline & |||| Sbjct & & 137 \\
\hline $\mathrm{GAC} A$ & CGATTACTAGTGATTCCAACTTCATGAAGTCGAGTTGCAGACTTC & 196Query & 121 \\
\hline AATC & TTAGCTTTTTAAGTTTCGCCATGTATCACTACAAAGCTTCTCTTTG & & 180 \\
\hline & |Sbjct & & 197 \\
\hline AATC & ITAGCTTTTTAAGTTTCGCCATGTATCGCTACAAAGCTTCTCTTTG & 256Query & 181 \\
\hline TACT & CACGTGTGTAGCCCAGATCATAAGGGGCATGATGACTTGACGTCA & & 240 \\
\hline & ||Sbjct & & 257 \\
\hline TACT & CACGTGTGTAGCCCAGATCATAAGGGGCATGATGACTTGACGTCA & 316Query & 241 \\
\hline TCCO & ГАСТСТTCGTAGGCAGTTTCATTAGAGTCCCCAACTTAATGATGG & & 300 \\
\hline & |||Sbjet & & 317 \\
\hline TCCC & ГACTCTTCGTAGGCAGTTTCATTAGAGTCCCCAACTTAATGATGG & 376Query & 301 \\
\hline $\mathrm{CAAC}$ & iGTTTCGCTCGTTGCGGGACTTAACCCAACATCTCACAACACGAGC & & 360 \\
\hline & ||||Sbjct & & 377 \\
\hline CAAC & iGTTTCGCTCGTTGCGGGACTTAACCCAACATCTCACAACACGAGC & 436Query & 361 \\
\hline TGTC & ACCACCTGTCTCTCGGTTCCCGAAGGCACAAGTATACTTCTATAC & & 420 \\
\hline & ||||Sbjct & & 437 \\
\hline TGTC & ACCACCTGTCTCTCGGTTCCCGAAGGCACAAG & 496Query & 421 \\
\hline
\end{tabular}

TCTCCCGAGGATGTCA 436

16S ribosomal RNA gene, partial sequence Length $=1468$ Score $=689$ bits $(373)$, Expect $=0.0$ Identities $=373 / 373(100 \%)$, Gaps $=0 / 373(0 \%)$

Query 3 CTTGCTAAATGGACTCATGGCGGACGGGTGAGTAACGCGTAAAGAACTTGCCCTTTAGAC

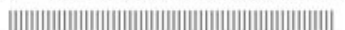

Sbjet 1396 CTTGCTAAATGGACTCATGGCGGACGGGTGAGTAACGCGTAAAGAACTTGCCCTTTAGAC Query 63 TGGGATAACAGAGGGAAACTTCTGATAATACTGGATAAGTTAGTATATCGCATGATATGC ||||||||||||||||||||||||||||||||||||||||||||||||||||||

Sbjct 1336 TGGGATAACAGAGGGAAACTTCTGATAATACTGGATAAGTTAGTATATCGCATGATATGC

Query 123 AAATGAAAGCTACGGCACTAAAGGAGAGCTTTGCGTCCTATTAGCTAGTTGGTAAGGTAA

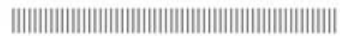

Sbjet 1276 AAATGAAAGCTACGGCACTAAAGGAGAGCTTTGCGTCCTATTAGCTAGTTGGTAAGGTAA

Query 183 GAGCTTACCAAGGCGATGATAGGTAGCCGGCCTGAGAGGGTGGACGGCCACAAGGGGACT ||||||||||||||||||||||||||||||||||||||||||||||||||||||||||||||||

Sbjct 1216 GAGCTTACCAAGGCGATGATAGGTAGCCGGCCTGAGAGGGTGGACGGCCACAAGGGGACT

Query 243 GAGATACGGCCCTTACTCCTACGGGAGGCAGCAGTGGGGAATATTGGACAATGGAGGAAA

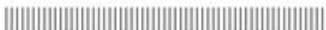

Sbjet 1156 GAGATACGGCCCTTACTCCTACGGGAGGCAGCAGTGGGGAATATTGGACAATGGAGGAAA

Query 303 CTCTGATCCAGCAATTCTGTGTGTGTGAAGAAGGTTTTAGGACTGTAAAACACTTTTAGT |||||||||||||||||||||||||||||||||||||||||||||||||||||||||| $\mid$

Sbjct 1096 CTCTGATCCAGCAATTCTGTGTGTGTGAAGAAGGTTTTAGGACTGTAAAACACTTTTAGT 1037

Query 363 AGGGAAGAAAAAA |||||||||||||

Sbjct 1036 AGGGAAGAAAAAA 
and bilateral salpingectomy by laparoscopic assistance. Antimicrobial therapy initially performed with lincosamide (clindamycin) plus Gentamycin was shifted to Tetracycline (100 mg x 2) and Metronidazole (500 mg x 2) for ten days, in accordance to microbiological supports. Treatment was completed with L-thyroxine, lansoprazole and enoxaparin for other clinical indications or on the basis of co-morbidity.

The patient recovered to normal either inflammatory indexes or physical exams, at controls on late January, mid February and June 2010.

\section{DISCUSSION}

In our case microbiological findings matched with clinical follow-up confirming anaerobe presence and pathogenic role. Up to date only 12 articles in English PubMed literature are available to specify Leptotrichia amnionii. Since its discovery in amniotic fluid by Shukla, et al in 2002 (14) as a novel bacterium, in the last ten years poor acquisition came by cultural and molecular methods $(3,8,10)$. Only a bit is known about its metabolism, pathogenic mechanisms and drug susceptibility, but recent evidence based studies show the role of Leptotrichia amnionii in bacterial vaginosis (9), urogenital tract infections (1), arthritis (7), endocarditis (2) and bacteremia (4). Furthermore the most recent immunological findings (12) suggest a cell mediated activation in host in accordance with epidemiological assessment of risk factors as pre term labor (5), organ transplantation (6) and HPV infections (13).

Anaerobes are somewhat neglected in routine analysis as if they were a minor field of microbiology, although the evidence of their remarkable superiority in quantity (10/100fold) versus aerobes in human habitats. Difficulties to collect, culture and preserve compel to time consuming and expensive efforts without the evidence of clinical efficacy; i.e. on the bases of few studies proven evidence, anaerobes investigation in paediatric blood culture usually isn't ever performed in routine protocols (11).

Identification at species level is sometimes impossible and susceptibility tests involving many genera (Leptotrichia included) lack of extensive standardization till nowadays (CLSI M11-A7 Vol.27 №2 - 2007; M11-S1, 2009)

This work has the aim to highlight how wide diagnostic tools genomics (and prospectively proteomics) can offer to ameliorate routine knowledge of anaerobes universe.

Is it the time to re-think their clinical impact, therapeutic options and drug-resistance?

\section{TRANSPARENCY DECLARATION}

The authors declare no commercial or conflict of interest in this work.

\section{REFERENCES}

1. Boennelycke M, Christensen JJ, Arpi M, Krause S. Leptotrichia amnionii found in septic abortion in Denmark. Scand J Infect Dis 2007; 4: 382-3.

2. Caram LB, Inefsky JP, Read KM, et al. Leptotrichia endocarditis: report of two cases from the International Collaboration on Endocarditis (ICE) database and review of previous cases. Eur J Clin Microbiol Infect Dis 2008; 27 (2): 139-43.

3. Carina M, Thilesen CM, Nicolaidis M, et al. Leptotrichia amnionii, an emerging pathogen of the female urogenital tract. J Clin Microb 2007; 45 (7): $2344-7$

4. De Martino J, Mashoudeau I, Brettes JP, Piemont Y, Monteil H, Jaulhac B. Peripartum bacteremias due to Leptotrichia amnionii and Sneathia sanguinegens, rare causes of fever during and after delivery. $J$ Clin sanguinegens, rare causes
Microbiol 2004; 12: 5940-3.

5. Di Giulio DB, Romero R, Amogan HP, et al. Microbial prevalence, diversity and abundance in amniotic fluid during preterm labor: a molecular and culture-based investigation. PLoS One 2008; 3 (8): e3056.

6. Domann E, Hong G, Imirzalioglu C, et al Culture-independent identification of pathogenic bacteria and polymicrobial infections in the genitourinary tract of renal transplant recipients. J Clin Microbiol 2003; 41 (12): $5500-10$

7. Goto M, Hitomi S, Ishii T. Bacterial arthritis caused by Leptotrichia amnionii. J Clin Microbiol 2007; 6: 2082-3.

8. Gundi VA, Desbriere R, La Scola B. Leptotrichia amnionii and the female reproductive tract. Emerg infect Dis 2004; 10: 2056-7.

9. Haggerty CL, Totten PA, Ferris M, et al. Clinical characteristics of bacterial vaginosis among women testing positive for fastidious bacteria. Sex Transmit Infect 2009; 85 (4): 242-8

10. Kwasniewska A, Nawrot R, Skoczynski M. Prevalence of Leptotrichia amnionii sp. nov. in pregnant women. Ginekol Pol 2006; 77 (7): 523-6.

11. Lee CS, Hwang B, Chung RL, Tang RB. The assessment of anaerobic blood culture in children. J Microbiol Immunol Infect 2000; 33: 49-52.

12. Marconi C, de Andrade Ramos BR, Peraçoli JC, Donders GG, da Silva MG. Amniotic Fluid Interleukin-1 Beta and Interleukin-6, but not Interleukin-8 Correlate with Microbial Invasion of the Amniotic Cavity in Preterm Labor. Am J Reprod Immunol 2011 Jan 9. doi: 10.1111/j.16000897.2010.00940. [Epub ahead of print].

13. Nawrot R, Kamieniarz K, Malinowska M, et al. The prevalence of Leptotrichia amnionii in cervical swabs of HPV positive and negative women. Eur J Gynaecol Oncol 2010; 31 (4): 425-8.

14. Shukla SK, Meier PR, Mitchell PD, Frank DN, Reed KD. Leptotrichia amnionii sp. nov., a novel bacterium isolated from the amniotic fluid of a woman after intrauterine fetal demise. Jour of Clin Microb 2002; 9: 3346-9. 